\title{
Infant use of relative motion as information for form: Evidence for spatiotemporal integration of complex motion displays
}

\author{
ROMY V. SPITZ and JOAN STILES \\ University of California, San Diego, La Jolla, California \\ and \\ RALPH M. SIEGEL \\ Center for Molecular and Behavioral Neuroscience \\ Rutgers University, Newark, New Jersey
}

\begin{abstract}
Previous studies of infants' ability to integrate and to utilize relative motion as information for form in the absence of structural cues have primarily involved motions that are uniform in rate, direction, and path within the form to be constructed. In the present study, we examined infants' ability to integrate relative motion information from motions that are nonuniform along these dimensions, and from this integrative process to construct a coherently rotating twodimensional form. Infants' ability to integrate nonuniform motion was measured with regard to their ability to discriminate the rotating form from a noncoherent control display containing the same absolute motions. The results showed that discrimination of the coherent and incoherent displays was not demonstrated until 7 months of age. Two additional experiments were conducted to rule out the possibility that this discrimination was based on the detection of local regions of coherence, rather than the perception of the global rotating form. In both experiments, the results did not support discrimination based exclusively on local cues alone. From the combined results of all three experiments, we conclude that infants demonstrate the capacity to integrate the information contained within nonuniform trajectories into a coherent structure by 7 months of age.
\end{abstract}

The ability to perceive motion greatly influences the way in which we interpret our visual experiences. Motion can provide information about aspects of an object's structure, such as its shape or substance, as well as information about the object's significance or potential for use. Motion also provides visual cues to aid the observer in navigation and permits an accurate judgment of depth and distance. Because it is such a rich source of information, it has been hypothesized that motion may play a vital role in the development of perceptual and cognitive abilities within the first year of life. Thus, by examining the development of motion perception within the early

We would like to thank Richard A. Andersen for the use of his laboratory, as well as all the parents and children for their participation and cooperation in this project. This work was supported by a grant from The John D. and Catherine T. MacArthur Foundation Network on the Transition from Infancy to Early Childhood, Grant 1 R23 HD2051201, by Grant 1 K04 HD00564-01 from the National Institutes of Child Health and Human Development, Department of Health and Human Services, and by grants from the University of California, San Diego, Academic Senate and Biomedical Research Fund awarded to J.S., as well as a Charles H. Revson Foundation Fellowship NINCDS NS-07467 awarded to R.M.S., who is affiliated with the Center for Molecular and Behavioral Neuroscience at Rutgers. Requests for reprints should be addressed to R. V. Spitz, Center for Molecular and Behavioral Neuroscience, Rutgers University, 197 University Ave., Newark, NJ 07102. postnatal period, one can achieve a better understanding of the potential informational tools that the infant brings to the task of learning about the world, as well as how the use of those tools is elaborated over the course of development.

It is well documented that even newborn infants are responsive to motion as an event. Studies of infant visual preference have consistently shown that infants prefer moving as opposed to stationary displays (e.g., McKenzie \& Day, 1976; Sherrod, 1979) and attend longer to rapid motions than to slower motions (Ivinskis \& Finlay, 1980; Kaufmann, Stucki, \& Kaufmann-Hayoz, 1985). Such preferences for motion may be observed across a broad range of velocities. In fact, when results from many studies are combined, one finds infants within the first 5 months responding to moving patterns and objects for velocities from $44^{\prime}$ of arc $/ \mathrm{sec}$ to $118^{\circ} / \mathrm{sec}$ (Aslin \& Shea, 1990; Bertenthal, Bradbury, \& Kramer, 1989; Kaufmann et al., 1985; Wattam-Bell, 1990).

Taken as a whole, these studies document that even in early periods of development, infants respond to the movement of single objects. Yet the visual world that we experience seldom involves single objects in motion. Instead, there are usually multiple objects, or parts of objects in motion, all of which have the potential to participate in a variety of inter- and intraobject relationships. One rela- 
tionship that has been studied in both adult and infant perception is that of relative motion: the movement of one object or part of an object with respect to another. For infants, like adults, relative motion may serve a variety of functions. It may influence general arousal or highlight salient features of the display that might otherwise go unnoticed. For example, the relative motion of two objects may emphasize the respective edge boundaries of each object. In neither of these two roles does the relative motion provide any new information. Rather, it acts to recruit or direct the observer's attention to information that is already present in the display. For the adult perceiver, however, relative motion serves a more important function. It provides powerful cues that can be used both to parse the visual array into a set of rudimentary parts (e.g., lines or contours) and to integrate these parts into coherent wholes, such as surfaces or objects. The production of these cues comprises the third and fourth roles of relative motion, specifically those of segmentation and integration. Relative motion can produce cues for segmenting the visual array by defining boundaries that are not perceivable in the static array, as, for example, on the basis of differential motion. Relative motion can also provide cues for integrating separate aspects of the visual array. The role of integration is orthogonal to that of segmentation in the sense that, rather than serving to create or define inter- or intraobject boundaries, relative motion can act to link together parts of the array that share common motion features. For example, elements of the array that move together are likely to be integrated into one surface or to be perceived as connected to the same surface or object.

Studies of infant perception of relative motion have generally approached the developmental question in one of two ways. The first is to examine the influence of relative motion on infants' ability to distinguish visible contours. The second is to examine infants' ability to use relative motion to construct objects and surfaces in the absence of structural cues provided by visible contours. The use of the first approach has indicated that by 4 months of age infants can use relative motion cues in conjunction with the information provided by static contours, to assist in detecting the figural properties of surfaces. One demonstration of this ability comes from a series of studies done by Kellman, Spelke, and their colleagues (Kellman, Gleitman, \& Spelke, 1989; Kellman \& Short, 1985; Kellman \& Spelke, 1983; Kellman, Spelke, \& Short, 1986; Spelke, 1988). In their original work, a group of 4-month-old infants were presented with a display consisting of a long, thin rod whose center was hidden behind a block (Kellman \& Spelke, 1983). The infants were placed in one of three display conditions such that the rod remained stationary behind the block, the rod and block moved together as a unit, or the visible ends of the rod shared a common motion path behind the stationary block. The results indicated that, unlike adults who perceived the rod as a unitary or continuous object in all three of the conditions, the infants did so only when the two rod fragments moved together while occluded by the stationary block. We can conclude from Kellman and Spelke's results that relative motion can help young infants to distinguish the differently moving pieces of a contour array. The findings indicate, at the very least, that relative motion can serve to emphasize salient aspects of the display. ${ }^{1}$

The motion display assisting the infants' detection of the unitary rod in Kellman and Spelke's study is additionally interesting in that it actually involves a combination of relative motion cues. Specifically, the two rod fragments moved together along the same motion path (i.e., common motion, one type of relative motion), yet their motion contrasted with the stationary block (differential motion, a second type of relative motion). This suggests that at least one of the cues, if not both, can be used by the perceptual system of young infants. However, only particular types of relative motion seem able to induce the perception of continuity for young infants. For example, if the display contained contrasting relative motion whereby the two rod pieces moved in different directions, similar to the motion of a "see-saw" (Kellman \& Short, 1985), or if the block alone was moved (Kellman \& Spelke, 1983), then the 4-month-olds did not perceive the rod as unitary. On the basis of these studies it can be concluded that at as early as 4 months of age, infants can detect the relative motion of a few contours, and, more importantly, they can use this relative motion to disambiguate the visual array and segregate differently moving contours.

Although also suggesting that infants can use relative motion in its integrative function, the studies by Kellman and Spelke do not directly address this issue. Granrud et al. (1984) have provided more direct evidence of infants' ability to integrate motion cues on the basis of relative motion, in their work on infant perception of kinetic information for depth such as accretion and deletion of texture. Granrud et al. have examined infants' ability to use such information in motion displays that do not contain pictorial information for depth order. They have done so by presenting 5- and 7-month-old infants with computergenerated displays consisting of large, randomly placed dots on a uniform background. The information for spatial layout in these displays is given by a combination of relative motion cues and changes in texture. Each dot in the display was designated as belonging to one of two surfaces, where all the dots assigned to a given surface moved in the same direction at a uniform speed. Between the two surface groups, the speed of motion was the same, but the dot motion was in the opposite direction. When the dots were set in motion, the display appeared to consist of two moving surfaces, with one surface passing in front of (i.e., occluding) the other. The perception of the two independently moving surfaces is the result of extracting relationships between the motions of various dots within a given area of the display. Dots that share a particular motion path (i.e., that move in the same direction) will tend to be grouped together to form larger regions of com- 
mon motion. At the same time, dots occupying nearby locations that have contrasting motion paths will be segregated from one another to create clear boundaries between the two apparent surfaces. In conjunction with this integration and segmentation based on relative motion, the systematic onset and offset of dots designed to simulate accretion and deletion of texture can be used to indicate the relative ordering of the surfaces in depth. It is important to note that these apparent surfaces were perceivable only when the dots were in motion. The static version of the display appeared as a single flat array of dots with no visible contours present to aid the subjects.

The results from Granrud et al. (1984) demonstrate that by 7 months of age infants could use the segmentive and integrative properties of relative motion, as well as changes in visual texture as information for form and depth-order. Specifically, the 7-month-olds preferentially reached for the nearer of the two apparent surfaces. The 5-month-olds as a group did not systematically show the same reaching preference. Infant perception of the surfaces themselves was not measured directly. Consequently, it may be the case that the 5-month-olds did perceive the two surfaces (i.e., they could integrate on the basis of relative motion) but were unable to use the texture cues for ordering in depth. In either case, this study provides strong evidence that infants are capable of using relative motion to construct forms and surfaces in the absence of static cues to form (e.g., visible contours) by at least 7 months of age.

The studies discussed thus far have involved the construction of form from the relative motion of either real figures (e.g., Kellman \& Spelke, 1983) or one group of dots relative to another group of dots (Granrud et al., 1984). In both cases, the type of motion described by the form has been translation. Consequently, all the points on the to-be-constructed form have moved not only in the same direction, but also at the same speed and along identical linear paths. The studies indicate that early in the postnatal period infants can use the integration of such uniform motions to construct surfaces. But are the infants' integrative abilities limited to uniform motions during this period? Or are they able to integrate more complex sets of motion trajectories? There is some indication that the integrative abilities and use of relative motion information might be limited to translation for 4-month-olds (Kellman \& Short, 1985; see note 1). If this is true, the kind and degree of information provided by relative motion would be highly constrained in early infancy and unlike the integrational use of relative motion accomplished by the adult perceptual system. The visual world contains objects that move in a variety of ways other than translation. For a form undergoing many of these motions, the different points on the form's surface might follow a similar trajectory, but not necessarily the same motion path. Consider, for example, a disk undergoing rotation. The points on the disk's surface lying nearest to the edge would travel farther and faster than points near the disk's center, resulting in a different (i.e., nonuniform) motion path for points in the two positions within a given time interval.
Yet the arcs described by the motion of points in the two positions would be related; they would be arcs along the same central angle. ${ }^{2}$

For the developing visual system of the infant, the integration of nonuniform motions into a global or coherent form may pose a particularly difficult integrative problem. Not only must infants perform the integration from the motion of isolated points, they must do so by detecting the relationships between points undergoing motions that vary in both speed and the precise path taken. The problem itself seems formidable, yet the mature visual systems of both human and nonhuman primates do this with ease (Siegel \& Andersen, 1988, 1990). In the present study, we investigated when young infants come to perform the type of sophisticated integration that will enable them to use nonuniform motions as information for object shape in the absence of other structural cues. This goal was accomplished through the use of a habituationof-looking procedure to assess infants' ability to discriminate between two displays, each consisting of 128 points of light (i.e., dots) moving within a circular area. The displays used in this study are based on those used by Siegel and Andersen in their work with human adults and rhesus monkeys. In one display, the coherent rotation display, the dots appeared as if they were points lying on the surface of a rotating disk. In the other display, the incoherent display, the same set of motion paths that produced the perception of a rotating disk were reordered so that the dots appeared to be moving randomly within the confines of the circular display area. Each dot in the coherent and incoherent displays was programmed to follow a specific trajectory for a limited time period $(560 \mathrm{msec})$. The inclusion of these features ensured that the motion trajectory described by any one dot provided only a limited amount of information. Thus the perception of the rotating form required the perceiver to integrate the motion information across many points. Because of this, these rotational coherent motion displays offered a powerful test of the infant's spatiotemporal integrative abilities. This specific set of displays provided a particularly well-controlled test of integrative abilities in that the coherent displays and their incoherent controls were created in such a way that they were equated for other cues to structure, such as changes in texture density or luminance, as well as changes in nonstructural cues, such as the number of points, the duration of each point, and the motion of individual trajectories. As a result, the coherent and incoherent displays differed only in the presence or absence of the rotating form-a form that could only be perceived through the integration of the individual, nonuniform trajectories across the display.

\section{EXPERIMENT 1}

\section{Method}

Subjects. A total of 36 full-term infants participated in this experiment. Six males and 6 females were assigned to each of the following groups: 4-month-olds $(M=16.1$ weeks, $S D=3.4$ days $)$, 5-month-olds ( $M=20.3$ weeks, $S D=2.1$ days), and 7 -month- 
olds ( $M=28.1$ weeks, $S D=4.3$ days). An additional 7 infants failed to complete testing, because of fussing or failure to habituate. Within each age group, the infants were randomly assigned to one of two experimental conditions, with the constraint that equal numbers of males and females were assigned to both conditions. The participants were recruited through advertisements placed in local papers, and they were predominately of middle socioeconomic status.

Stimuli. Two stimulus displays were used in the present experiment, one coherent and one incoherent. Both appeared as a set of 128 moving points of light confined within a $314-\mathrm{cm}^{2}$ circular area. Each point was $6.69 \mathrm{~mm}$ in diameter, and the entire display subtended $36.87^{\circ}$ of visual angle. In the incoherent display, the motion of the points appeared completely random, whereas in the coherent display, the motion of the points appeared as if they were points on the surface of a disk rotating clockwise about an axis perpendicular to the display surface.

Each display was computer generated and then videotaped for presentation. To generate the stimuli, first a circular area of space was designated on a CRT screen with a refresh rate of $40 \mathrm{~Hz}$. Then the 128 points were randomly placed within this area, with the constraint that the points be equally distributed across the display. Once the points were placed, a motion pathway was calculated for each point. The pathway simulated the motion that the point would follow if it was actually on the surface of a rotating disk. On the basis of its computed motion path, each point was assigned a motion trajectory that defined the rate and direction of motion as well as the path. The rate of point motion varied, with points on the periphery of the disk moving more rapidly than points located more centrally. Each point appeared to move smoothly from the beginning to the end of its assigned path. Although it is difficult to convey the actual rotation through the use of a static picture, Figure 1 will give the reader an idea of the display's appearance. For adults, the visual effect of the coherent display is that of a set of points lying on the surface of a moving disk.
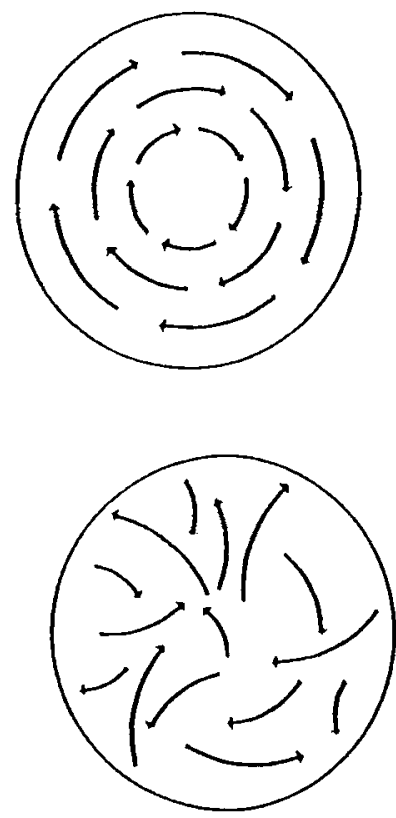

Figure 1. The coherent (top) and incoherent (bottom) motion displays. This figure is intended to depict the appearance of the coherent and incoherent displays. It is not intended to be an exact rendering of how the display was structured in the coherent display and reordered in the incoherent display.
The incoherent displays were derived directly from the coherent displays. Generation of the displays began with the same motion trajectories that were computed for the coherent displays. The difference came with the assignment of motion trajectories to the location of points in the display. The display was divided horizontally into a series of narrow strips, with the length of each strip equal to the width of the display. The trajectories assigned to the points located in each strip were randomly reassigned. Thus, the motion trajectory might have been assigned to a point occupying the same position as it had in the coherent display (i.e., its correct placement on the disk) or to a point located at the opposite end of the strip. The perceptual result was an incoherent motion display in which points moved randomly within the circular confines of the display area. The important point is that the reassignment of trajectories produced a situation in which the individual arc trajectories were identical for the coherent and incoherent displays, yet the spatial structure that permitted the perception of the global rotating form was destroyed.

Another important feature of the displays was the limited duration of each point. As in the original work done by Siegel and Andersen $(1988,1990)$, each of the 128 points in the display had a point duration of $560 \mathrm{msec}$. That is, a point would appear in the display and would take $560 \mathrm{msec}$ to travel across an area of the CRT screen to its final destination. Furthermore, the onset and offset of the point lights were staggered for both the coherent and the incoherent displays. Points appeared, followed their motion path, and then disappeared, but did so at random intervals, so that no periodicity in the display was detectable. The removal of periodic changes eliminated the possibility that infants could discriminate the coherent and incoherent displays by restricting their attention to any particular points or pairs of points. Therefore, within any time interval, the array of points was not simply rotated in space and repainted on the screen. Rather, it was transformed into a new array as some points appeared in the array and began their motion paths while others disappeared as they reached the end of their 560msec lifetime. In addition, each habituation and test display was constructed from a different set of initial points to prevent the infants from relying on any particular motion pattern as a means for discrimination. Across the displays (coherent and incoherent), the number of points (128), point size $(6.69 \mathrm{~mm})$ and duration (560 msec), absolute motions, and point density were identical. The values for each of these parameters were selected because they were found to be the optimal values for discrimination between coherent rotation and incoherent motion by adult humans and rhesus monkeys (Siegel \& Andersen, 1988, 1990).

Apparatus. The infants were individually tested in a large room containing a looking chamber designed after the one used by Bertenthal, Proffitt, and Cutting (1984). This chamber was arranged so that the infant, seated on the parent's lap, faced a half-silvered mirror placed at a $45^{\circ}$ angle from the infant's line of sight. With the display monitor positioned at a $45^{\circ}$ angle from the mirror, a reflected image of the stimulus displays could be presented directly in front of the infant, while an unobstructed view of the infant's face and eyes was still permitted. The portion of the mirror not required to view the displays was covered with a black matte screen to eliminate reflections that might otherwise distract the infant. The infant's face was illuminated by a small $40-\mathrm{W}$ light attached to the upper outside portion of the chamber. This light provided the required lighting for the camera. An opaque screen extended $19 \mathrm{~cm}$ from the top of the chamber to just above the infant's head to ensure that the parent could not see the displays. The infant's eye movements were videotaped through the half-silvered mirror using a lowlight-sensitive video camera. This camera, as well as the video equipment for viewing the infant, was behind the chamber and out of the infant's sight. The experimenter remained behind the chamber during the actual testing.

Design and Procedure. The infants were randomly assigned to one of two experimental conditions. In Condition 1, the incoher- 
ent displays were used for the habituation trials and the coherent displays were used for the test trials; in Condition 2, this order was reversed. Because of technical constraints limiting the maximum length of each trial to $8 \mathrm{sec}$, a fixed-trials habituation procedure was used. A preliminary study determined that the optimal number of trials required for habituation was 23 .

A typical session was conducted as follows: the infant was brought into the testing room and allowed approximately $15 \mathrm{~min}$ to become familiarized with the room. During this time, the experimenter explained the procedure to the parent and requested that the parent refrain from interacting with the child during testing. The infant was then seated on the parent's lap $38 \mathrm{~cm}$ from, and directly facing, the looking chamber. When the infant appeared to be reasonably still and was looking forward, the experimenter started the camera and presented the stimuli in the following test sequence. Each infant was first shown 23 consecutive trials of either the coherent or the incoherent display, depending on the assigned condition (habituation phase); these were followed by 5 trials of the opposite display (test phase) and then by two 8 -sec posttest trials. The posttest trials consisted of static pictures of a lightly colored red and blue train, and they were used in the assessment for infant fatigue. Between trials there was a 4-sec intertrial interval, during which the screen was darkened. Both the onset and the offset of each trial were signaled by an audible click, the purpose of which was to encourage infant orientation to the stimulus display.

Data reduction. The visual behavior of the infant was scored from videotape by one experimenter for the total fixation time for each trial. Within each of the 8-sec trials, multiple fixations were scored if the infant looked away from the display and then returned to reexamine it before the termination of the display. All fixations within a trial were then summed to achieve the total fixation time for each trial. A second scoring was performed on one third of the testing sessions by a different individual who was blind to the purpose of the experiment as well as to the age and experimental condition of the child. Interrater reliabilities were 0.96 , with the criterion that the amount of looking for each of the 30 trials had to agree within $1 \mathrm{sec}$ of the original scoring. The measure for the assessment of habituation was based on a comparison of the mean fixation time for the first and final three trials of the habituation display. The measurement for the assessment of dishabituation was based on a comparison of the mean fixation time for the final three habituation trials with the mean fixation time for the first three trials of the test display. The measure for the assessment of fatigue was based on a comparison of the mean fixation time for the final three trials of the habituation display with the mean fixation time for the two posttest displays.

\section{Results}

The results of this experiment revealed that 7 -montholds, but not 4- or 5-month-olds, dishabituated to the test stimulus, indicating that they could discriminate the coherent and incoherent displays. Discrimination at 7 months was not affected by order of stimulus presentation or gender. These findings were verified by a $3 \times 2 \times 2 \times$ 3 (age $\times$ gender $\times$ condition $\times$ trial block) mixed design analysis of variance (ANOVA). The between-subject factors were age $(4,5$, and 7 months), gender (male and female), and condition (incoherent-coherent and coherentincoherent). The within-subject factor was trial block (first habituation trials, final habituation trials, and test trials). The results of the ANOVA showed a main effect of trial block $[F(2,48)=71.64, p<.001]$. There were no main effects for age $[F(2,24)=1.60]$, gender $[F(1,24)=$ $0.00]$, or condition $[F(1,24)=0.13]$. The age $\times$ trial

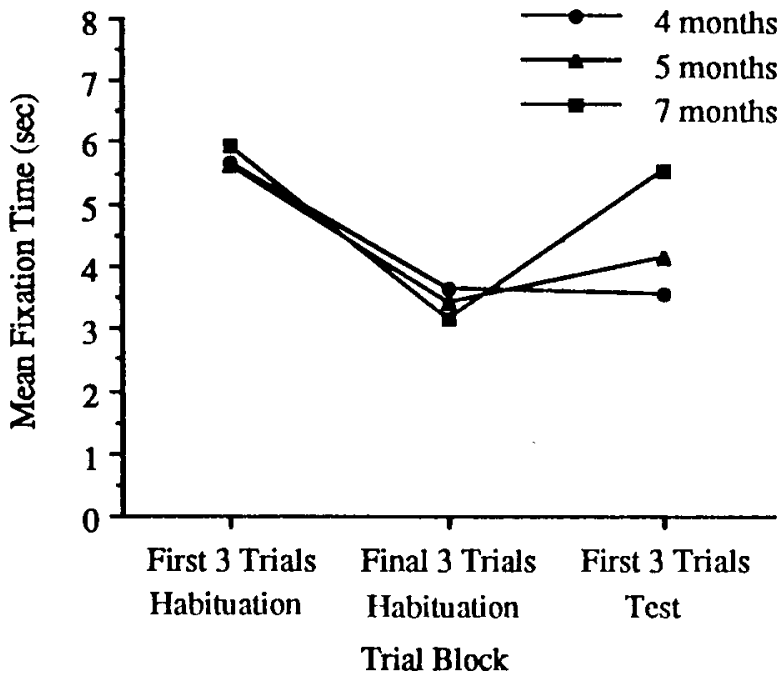

Figure 2. Mean fixation times in response to the habituation and test displays, plotted by age.

block interaction was significant $[F(4,48)=7.14, p<$ $.001]$. There were no other significant interactions.

The major results of this study can be seen most clearly in the age $\times$ trial block interaction illustrated in Figure 2 . Simple effects analysis for age showed a main effect for trial block at every age $[F(1,24)=33.12,31.60$, and 380.99 , for the 4-, 5-, and 7-month-olds, respectively, $p<.001$ for each age]. Multiple comparisons were made between trial blocks within each age group, employing a Tukey $H S D$ procedure with a significance criterion of $p<.01$. The analysis confirmed the pattern of changes in fixation illustrated in Figure 2. Four-month-olds demonstrated a significant decrease in mean fixation time from the first $\left(M_{\text {First }}=5.66 \mathrm{sec}, S D=0.93\right)$ to the final trials of the habituation displays $\left(M_{\text {Final }}=3.63 \mathrm{sec}, S D=\right.$ 0.73 ), but no recovery in response to the test display $\left(M_{\text {Test }}=3.55 \mathrm{sec}, S D=1.01\right)$. At 5 months, a similar decrement was evident during the habituation phase $\left(M_{\text {First }}=5.63 \mathrm{sec}, S D=1.01 ; M_{\text {Final }}=3.40 \mathrm{sec}\right.$, $S D=1.06)$, and although there was a small amount of attentional recovery at test $\left(M_{\text {Test }}=4.12 \mathrm{sec}, S D=\right.$ 0.96 ), this increase was not significant even at $p<.05$. Seven-month-olds demonstrated a similar response decrement in response to the habituation stimulus $\left(M_{\text {First }}=\right.$ $\left.5.94 \mathrm{sec}, S D=0.99 ; M_{\text {Final }}=3.16 \mathrm{sec}, S D=1.28\right)$, but showed significant recovery in response to the test stimulus $\left(M_{\text {Test }}=5.51 \mathrm{sec}, S D=1.18\right)$.

A simple effects analysis for trial block showed no effect for age at either the first habituation trials $[F(2,24)=$ $0.37]$ or the final habituation trials $[F(2,24)=0.50]$, but did confirm a significant effect for the test trials $[F(2,24)=9.12, p<.001]$. Multiple comparisons between ages were conducted, employing a Tukey $H S D$ procedure; the analysis showed no between-age differences in the amount of looking for either the first or final trials of the habituation display. However, on the test trials, 7- 
month-olds looked significantly longer than did either 4month-olds $(p<.01)$ or 5 -month-olds $(p<.05)$, who did not differ.

A separate one-way ANOVA was conducted to assess whether these results were attributable to fatigue on the part of the younger age groups. The mean fixation time for the final habituation trials was compared with the mean fixation time for the posttest displays. The analysis showed a significant effect of trial $[F(1,24)=7.94, p<.01]$, indicating that the younger two age groups were able to discriminate between the habituation display and the posttest display. Thus, the failure of the younger infants to discriminate between the habituation and test displays was not attributable to either inattention or fatigue, as is evidenced by the similar habituation patterns found across the three age groups and the significant attentional recovery demonstrated by these infants in response to the posttest display.

\section{Discussion}

The results of this experiment show that although all three age groups demonstrated very similar habituation patterns, only the 7-month-olds increased the time spent in visually examining the displays in response to the presentation of the novel test display. These results suggest that somewhere between the ages of 5 and 7 months of age, infants come to be able to integrate complex, nonuniform dynamic patterns into a well-structured whole. However, as Siegel and Andersen (1988) point out in their original paper, there is an alternative way for the infant to discriminate the coherent and incoherent displays which does not rely on their perception of a coherent form. Instead, the infant might discriminate the displays on the basis of local parallelisms that are inherent in the coherent display. Local parallelisms refer to transient relative motion cues whereby two points appear to be moving along parallel paths. Thus, instead of detecting the global form (the rotating disk), the infants may simply be detecting the presence of similarities in the dots' trajectories or locally coherent motion. The existence of similar trajectories at nearby points in the visual field permits the visual system to detect the movement of surfaces and objects. Yet detecting the sudden presence or absence of such local events is not identical to the perception of global surface motion. There is sufficient evidence for the early perception of uniform vertical coherence under optimal stimulus conditions to make this possibility a valid concern (Spitz \& Kleffner, 1990, 1992).

To ensure that their subjects were not discriminating on the basis of local cues, Siegel and Andersen (1988, 1990) devised a control procedure in which they masked $96 \%$ of the display by area, leaving a small window through which a limited portion of the display ( $4 \%$ by area) was visible. The masking reduced the number of trajectories below that necessary for the perception of the rotating form but was sufficient for the production of local parallelisms. ${ }^{3}$ The authors reasoned that if subjects were able to use the available local cues to discriminate the unmasked displays, the masked displays should be as easily discriminable. Siegel and Andersen report that both their human and their monkey subjects were able to discriminate the displays when they were masked in this fashion. However, the subjects' thresholds for making the discrimination were much higher under the masked condition than under the unmasked condition, reflecting an impairment in performance. This impairment in performance suggests that the subjects' ability to discriminate the standard, unmasked displays was not based principally on local relative motion cues. To test the possibility that the 7-month-olds in the present study were using these local cues to discriminate the displays rather than the perception of the rotating form, we modeled our second experiment after the masked control procedure used by Siegel and Andersen.

\section{EXPERIMENT 2}

The purpose of this experiment was to examine whether infants could discriminate the coherent motion displays from the incoherent ones on the basis of local parallelisms alone. As previously mentioned, these involve small areas of common motion that are present in the rotating coherent displays. A control procedure modeled after that of Siegel and Andersen $(1988,1990)$ was constructed, in which infants viewed only the central $4 \%$ by area of each display. If infants were capable of discriminating on the basis of local cues, they should have been able to differentiate these simplified displays. A separate group of 10 adult subjects were also presented with these masked displays in order to determine whether the small size of the field adequately permitted the perception of local parallelisms. Eight of the 10 adult subjects were able to discriminate between the coherent and incoherent displays, although only 1 of those 8 was able to correctly identify the motion as a rotation. This result indicates that the masked displays were of sufficient size to present the local parallelisms, but did not include enough of the motion cues for the subjects to identify the rotating structure.

\section{Method}

Subjects. A total of 8 full-term 7 -month-olds ( $M=28.1$ weeks, $S D=1.99$ days) participated in this study. An additional 5 infants failed to complete testing, because of fussing or failure to habituate. Each infant was randomly assigned to one of two experimental conditions, with the constraint that equal numbers of males and females were assigned to both conditions.

Stimuli and Apparatus. In constructing the stimuli for the present study, we began with displays identical to those in Experiment 1. A black matte screen was then placed over each display so that only $4 \%$ of the display by area was visible to the infant. This portion, which was located slightly left of the center of the display, appeared as a square (area $=14.52 \mathrm{~cm}^{2}$ ) in which the points of light corresponding to either the coherent or the incoherent displays appeared. At any moment during the 8-sec display, there was an average of $6.39(S D=2.45)$ points present and moving within that area. Thus, the number of points was sufficient to permit the detection of local parallelisms, but it was below that needed for the perception of the rotating form. The apparatus used in this study was identical to that used in Experiment 1. 
Design and Procedure. The design and procedure used in this study were identical to those described for Experiment 1, with the exception that fewer habituation trials were given to each infant. A preliminary study conducted to determine the number of trials necessary to achieve habituation showed that the masked displays were apparently less interesting to the infants, and only 10 habituation trials were required. The data reduction procedure used in this study was identical to that in Experiment 1 with the exception that, owing to the fewer number of habituation trials, the mean fixation time used to assess habituation, dishabituation, and fatigue involved an average of 2 rather than 3 trials. Interrater reliability was 0.94, using the same criterion as that in Experiment 1.

\section{Results and Discussion}

The results of this study showed that 7-month-olds did not show the attentional recovery in response to the test display necessary to demonstrate discrimination. Thus, it does not appear that infants can discriminate the masked displays by using local parallelisms alone. These data were analyzed with a $2 \times 2 \times 3$ (gender $\times$ condition $\times$ trial block) mixed design ANOVA. The between- and withinsubject factors were identical to those described for Experiment 1 , with the exception that there was no factor of age in the present study. The results showed a main effect of trial block $[F(2,8)=51.26, p<.001]$. There were no other main effects or interactions. The main effect of trial block is presented in Figure 3. Multiple comparisons were conducted between trial blocks, using the same procedure and criterion described for Experiment 1. The infants demonstrated a significant decrease in mean fixation time from the first to the final trials of the habituation display $\left(M_{\text {First }}=5.01 \mathrm{sec}, S D=0.98 ; M_{\text {Final }}=\right.$ $2.84 \mathrm{sec}, S D=0.60$ ); however, no response recovery to the test display was found $\left(M_{\text {Test }}=1.95 \mathrm{sec}, S D=\right.$ 1.18). As in Experiment 1, a separate ANOVA was conducted to assess infant fatigue. This analysis showed a main effect of trial $[F(1,5)=10.72, p<.05]$. The in-

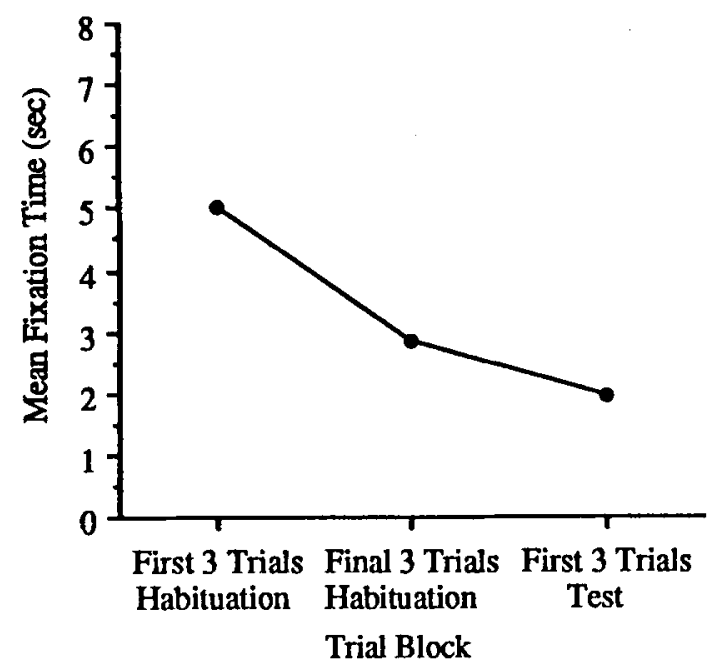

Figure 3. Mean fixation time in response to the habituation and test masked displays. fants did discriminate the habituation displays from the posttest displays, indicating that the lack of response recovery in response to the masked displays was not due to infant fatigue.

\section{EXPERIMENT 3}

Experiment 2 provided one means of testing the possibility that 7-month-old infants had discriminated the test displays on the basis of local parallelisms. The results showed that 7-month-olds did not discriminate on the basis of parallelisms available from a local region containing an average of six dots. It is possible, however, that infants are only able to detect the relative presence of local parallelisms when either larger motion fields or more points are involved. Thus, the discrimination evidenced by the infants during Experiment 1 might still be explained by the perception of local cues without recourse to the perception of rotational global motion. The purpose of Experiment 3 was to provide a different approach to assessing whether the 7-month-olds were simply detecting in response to the presence as opposed to the absence of local coherence. ${ }^{4}$ This was accomplished by testing infant discrimination of two coherent motion displays that both contained local parallelisms: the rotating disk display and a horizontally translating version of the same random-dot array. If infants were responding only to the appearance or disappearance of local parallelisms, they would not have been expected to discriminate between the two coherent displays.

\section{Method}

Subjects. A total of five 7-month-olds, 3 males and 2 females, participated in this experiment $(M=28.2$ weeks, $S D=3.1$ days).

Materials and Procedure. The materials and procedure used in this experiment were identical to those in Experiment 1, with the exception that instead of the coherent and incoherent motion displays, the infants were presented with the coherent rotation display during the habituation phase and a coherently translating version of this display during the test phase of the experiment. Interrater reliability was 0.98 .

\section{Results and Discussion}

The main finding of this experiment was that 7-montholds discriminated the two types of coherent motion displays. The results of a $2 \times 2$ (gender $\times$ trial block) mixed design ANOVA revealed a main effect of trial block $[F(2,6)=33.60, p<.001]$. There were no other significant main effects or interactions. The mean fixation time during each phase of the experiment is presented in Figure 4. Multiple comparisons between trial blocks were conducted with the same $p<.001$ criterion for significance as described for Experiment 1. These comparisons confirmed both the decrease in fixation from the first to the final trials of the habituation phase and a significant recovery of attention at test. These findings indicate that 7-month-old infants are capable of responding to more than simply the presence as opposed to the absence of parallelisms. 


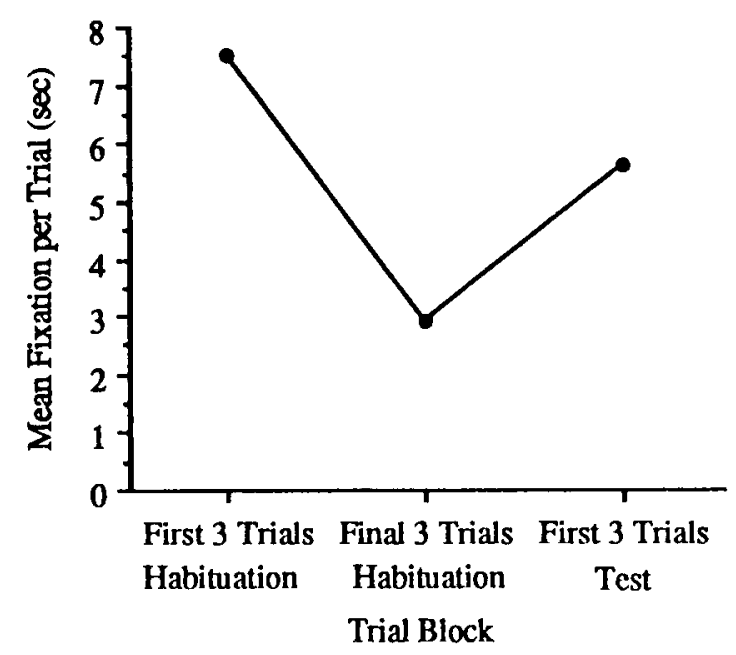

Figure 4. Mean fixation time in response to the global rotation (habituation) and global translation (test) displays.

\section{GENERAL DISCUSSION}

Previous studies have revealed that, even very early in development, infants have the ability to perceive and use relative motion as information for form. In general, these studies have demonstrated that by 4-7 months of age, infants can use relative motion to do both segregation and integration within a visual array. There was also some suggestion that this early ability might be limited to translations for which the elements of the array move at similar speeds along identical paths. The purpose of the present series of experiments was to establish when, in the developmental process, infants demonstrated an ability to integrate a set of nonuniform motions as required for the perception of the rotating form. The results of the study show that this ability was present by 7 months of age, but it was not observed in infants in the younger two age groups. Specifically, 7-month-olds, but not 4- or 5-montholds, were able to discriminate reliably between the coherent rotating display and its incoherent control display.

In constructing the original coherent and incoherent displays, care was taken to equate them for factors such as the number, density, and duration of dots, as well as the absolute motions present in each display. Because of these features, and because of the fact that each dot's trajectory provided only a limited amount of information regarding the overall structure, the observed discrimination could be accomplished in only one of two ways. The first, and less sophisticated, way to discriminate the displays is to do so by detecting similarities in dot trajectories or local parallelisms. Such similarities are an integral property of surface motion, and, accordingly, they are present in the coherent rotation display. The procedures used to remove the rotational structure in order to create the incoherent motion control also removed the local parallelisms. Experiments 2 and 3 were designed to in- vestigate whether the discrimination observed for the 7month-olds in Experiment 1 could be attributed to the detection of local parallelisms. In Experiment 2, this was accomplished through masking all but $4 \%$ of each display. The use of such a restricted portion of the motion field permitted a test of discrimination under conditions in which the available number of motion trajectories was sufficient for subjects to detect the presence or absence of local parallelisms, yet the number was below that necessary to perceive the structure of the rotating disk. The lack of any demonstrable discrimination in Experiment 2 suggests that 7-month-olds do not appear to be using these transient local motion cues as their primary basis for discrimination. In Experiment 3, infants' ability to discriminate between two coherently moving displays, both of which contained local parallelisms, was tested. The findings from this experiment demonstrated that 7 -month-olds are not limited to discriminating on the basis of the appearance and disappearance of local parallelisms. Given the combined results from both experiments, it is unlikely that the discrimination of the original coherent and incoherent displays can be explained through recourse to perceptual processes restricted to local motion.

The second, and more probable, means of discriminating the original set of displays is to do so by detecting a rotating structure that is present in the coherent displays, but not the incoherent displays. This structure cannot be perceived by examining the motion of single points or even pairs of points. Its perception requires the integration of motion trajectories across the display. This should not be taken to mean that the infant either requires or includes the trajectory of each point in the integration process. As with adult observers, it is quite possible that some proportion of the points would provide sufficient information for the infant to extract the form of the rotating disk from the coherent displays. The important point is that the 7-month-olds' ability to discriminate these displays indicates that they can integrate motion trajectories that are dissimilar in speed, direction, and path across a fairly large array.

The small amount of recovery at test shown by the 5month-olds in Experiment 1 suggests that this ability to integrate motion cues of this type may be somewhat flexible with respect to its time of emergence. One possibility is that by adjusting the display parameters we may be able to demonstrate infants' ability to integrate such nonuniform motions at somewhat earlier ages. The parameters of the present displays were selected because they were the optimal values for adult humans and rhesus monkeys, and it is of course possible that these parameters are not optimal for young infants. Thus, the next logical step would be to adjust the parameters of the coherently rotating displays in order to examine the impact of such manipulations on infants' ability to integrate nonuniform motions across an array.

As part of a larger picture, the present study clarifies the point in development at which infants can construct a coherent, two-dimensional form via a global integra- 
tive process involving a large set of nonuniform motions, and it further extends our knowledge of infants' ability to use relative motion as information for form or structure. From previous studies, it is known that by 4 months of age infants can detect relative motion and can use relative motion cues to assist in detecting the figural properties of objects in conjunction with the information provided by static contours (e.g., Kellman \& Spelke, 1983). The work of Kellman and Spelke has also shown that this early ability to use relative motion information does not extend to all types of relative motion. Specifically, the 4-month-old appears to be capable of using common motion in its integrative role, while remaining fairly insensitive to the information provided by contrasting relative motions. By 5-7 months of age, infants appear to be able to use both types of relative motion, and at least by 7 months of age the infants can use the relative motion information provided by these cues to construct two surfaces moving in opposite directions in the absence of static structural information (Granrud et al., 1984). The present study extends this developmental continuum to the use of common and contrasting motions in which the motion contained within the field is nonuniform in speed and path of motion, as well as in direction. Specifically, the data show that by at least 7 months of age, infants are sufficiently capable of integrating nonuniform motion trajectories across an array, in such a way that they can utilize this information to construct an apparently rotating disk.

From this continuum, it can be seen that the perception, integration, and utilization of even these seemingly uncomplicated dynamic cues is itself a multifaceted event. The ability to process relative motion does not emerge full-blown at any one point in infancy. Rather, it is a process that appears, at least in rudimentary form, during early infancy and subsequently becomes elaborated across the developmental period. The elaboration appears to proceed in a fairly orderly fashion from the earliest stages of using motion to distinguish a uniformly moving surface from its stationary background; to integrating and segmenting surfaces on the basis of motions that share a common speed and path, yet differ in direction; and then to integrating motions that are dissimilar along all three parameters into a regular, coherent form, such as a twodimensional disk. The question of what aspects of the visual system, specifically, are changing to produce this progression is difficult to answer. It is tempting to suggest that the maturation proceeds through the hierarchy of motion processing regions since the levels of motion processing in the hierarchy functionally match those of the developmental sequence. However, the period of infancy is a time of rapid development in virtually every aspect of vision, from the retina and its receptors to the cortical processing of spatial and temporal information. At the present time, there is simply not enough known about the development of the basic sensory and perceptual abilities that are involved in motion processing to say definitively which of these possible factors might be in- volved in, or might mediate, the changes we see occurring in the infant's ability to perceive and use motion as information.

What is known is that within the same domain of using motion as information for structure, the progression continues. Relative motion can also be used to construct higher order, three-dimensional forms, as is exemplified by the case of biomechanical motion. The phenomenon of biomechanical motion refers to a set of point-light displays in which lights appear to move as if placed on the head and major joints of a person walking. Studies of infant sensitivity to the information carried by these forms indicate that, at 3 months of age, infants can distinguish between these biomechanical motion displays and other displays, using low-level motion cues such as differences in the phase relations of the point lights whose motion creates the form of the walker (Bertenthal et al., 1984; Bertenthal, Proffitt, Spetner, \& Thomas, 1985). More importantly, by 9 months of age, infants can perceive the human walker form depicted by the relative motions of points in the display. In conjunction with the findings of the present study, the biomechanical motion studies describe a sequence of developmental events within the domain of integrating nonuniform motion information into unified forms. The present study has demonstrated that by 7 months, infants can integrate a set of dissimilar motions into a two-dimensional, coherent form, while the results of the biomechanical motion studies indicate that by 9 months, infants can go beyond this level of integration to create an articulating three-dimensional structure in the absence of static cues.

\section{REFERENCES}

AsLin, R. N., \& SHEA, S. L. (1990). Velocity thresholds in human infants: Implications for the perception of motion. Developmental Psychology, 26, 589-598.

Bertenthal, B. I., Bradbury, A., \& Kramer, S. (1989, April). Velocity thresholds in 5-month-old infants. Poster presented at the biennial meeting of the Society for Research in Child Development, Kansas City, MO.

Bertenthal, B. I., Proffitt, D. R., \& Cutting, J. E. (1984). Infant sensitivity to figural coherence in biological motion. Journal of Child Psychology, 37, 213-230.

Bertenthal, B. I., Proffitt, D. R., Spetner, N. B., \& Thomas, M. A. (1985). The development of infant sensitivity to biomechanical motions. Child Development, 56, 531-543.

Granrud, C. E., Yonas, A., Smith, I. M., Arterberry, M. E., Glicksman, M. L., \& Sorkness, A. C. (1984). Infants' sensitivity to accretion and deletion of texture as information for depth at an edge. Child Development, 55, 1630-1636.

IvinSKIS, A., \& FinLAY, D. C. (1980, April). Cardiac responses in fourmonth-old infants to stimuli moving at three different velocities. Poster presented at the biennial meeting of the International Conference for Infancy Studies, New Haven, CT.

Kaufmann, F., Stucki, M., \& Kaufmann-Hayoz, R. (1985). Development of infants' sensitivity to slow and rapid motions. Infant Behavior \& Development, 8, 89-98.

Kellman, J. P., Gleitman, H., \& SPelke, E. S. (1989). Object and observer motion in the perception of objects by infants. Journal of Experimental Psychology: Human Perception \& Performance, 13, 586-593. 
Kellman, J. P., Short, K. R. (1985, June). Infant object perception from motion information: The problem of rotation. Paper presented at the Third International Conference on Event Perception and Action, Uppsala, Sweden.

Kellman, J. P., \& Spelke, E. S. (1983). Perception of partly occluded objects in infancy. Cognitive Psychology, 15, 483-524.

Kellman, J. P., Spelke, E. S., \& Short, K. R. (1986). Infant perception of object unity from translatory motion in depth and vertical translation. Child Development, 57, 72-76.

KoENDERINK, J. J., \& VAN DOORN, A. J. (1976). Local structure of movement parallax of the plane. Journal of the Optical Society of America, 66, 717-723.

KoENDERINK, J. J., \& VAN DoORN, A. J. (1981). Exterospecific component of the motion parallax field. Journal of the Optical Society of America, 71, 953-957.

MCKenZIE, B. E., \& DAY, R. H. (1976). Infant attention to stationary and moving objects at different distances. Australian Journal of Psychology, 28, 45-51.

SHERROD, L. R. (1979), Social cognition in infants: Attention to the human face. Infant Behavior \& Development, 2, 279-294.

Siegel, R. M. \& ANDERSEN, R. A. (1988). Perception of three dimensional structure from two dimensional motion in monkey and man Nature, 331, 259-261.

Siegel, R. M., A ANDERSEN, R. A. (1990). Perception of structure from motion in monkey and man. Journal of Cognitive Neuroscience, 2 , 306-319.

SPELKE, E. S. (1988). Where perceiving ends and thinking begins: The apprehension of objects in infancy. In A. Yonas (Ed.), The develop ment of perception: Minnesota Symposium on Child Psychology (pp. 197-234). Hillsdale, NJ: Erlbaum.

SPITZ, R. V., \& KLFFner, D. (1990, April). Infant perception of coherent motion; The effect of spatial displacement. Paper presented at the biennial meeting of the International Conference on Infant Studies, Montreal
SPITZ, R. V., \& KLEFFNeR, D. (1992, May). Global motion integration and the breakdown of directional motion (dmax) between 2 and 7 months. Paper presented at the biennial meeting of the International Conference on Infant Studies, Miami.

WATTAM-BELL, J. (1990). The development of maximum velocity limits for direction discrimination in infancy. Perception, 19, 369

\section{NOTES}

1. The ability of young infants to use relative motion cues to integrate surfaces is not limited to horizontal translations; it also occurs for vertical translations and translations in depth (Kellman et al., 1986). However, the ability may be limited to translatory movements, since infants do not appear to perceive the rod as unitary under conditions of rod rotation (Kellman \& Short, 1985).

2. In this paper, the use of the terms uniformity or nonuniformity of motion is describing specifically the motion of the points on a surface and not the motion of the to-be-constructed object. In the present case of rotation, the motion of the whole is uniform in the sense that it contains a uniform curl component (Koenderink \& van Doorn, 1976, 1981). However, the motions that must be integrated to construct that curl are themselves not uniform; they describe different trajectories and move at different velocities.

3. Siegel and Andersen found that their subjects were unable to perceive the rotating form when fewer than 32 points were present in the display. See Siegel and Andersen $(1988,1990)$ for further discussion of parameter manipulations.

4. We would like to thank the anonymous reviewer who suggested Experiment 3.

(Manuscript received October 16, 1989; revision accepted for publication August 12, 1992.) 